The Astrophysical Journal, 687:976-985, 2008 November 10

(C) 2008. The American Astronomical Society. All rights reserved. Printed in U.S.A.

\title{
INCLINATION-DEPENDENT EXTINCTION EFFECTS IN DISK GALAXIES IN THE SLOAN DIGITAL SKY SURVEY
}

\author{
Cayman T. Unterborn and Barbara S. Ryden ${ }^{1}$ \\ Department of Astronomy, The Ohio State University, Columbus, OH 43210; unterborn.1@osu.edu, ryden@astronomy.ohio-state.edu \\ Received 2008 January 15; accepted 2008 July 14
}

\begin{abstract}
We analyze the $r$-band absolute magnitude and $u-r$ color of low-redshift $(z<0.06)$ galaxies in the Sloan Digital Sky Survey Data Release 6. Galaxies with nearly exponential profiles (Sloan parameter fracDeV $<0.1$ ) fall on the blue sequence of the color-magnitude diagram; if, in addition, these exponential galaxies have $M_{r} \lesssim-19$, they show a dependence of $u-r$ color on apparent axis ratio $q$ expected for a dusty disk galaxy. We create a subsample of bright exponential galaxies, with typical Hubble types Sbc and Sc; then, by comparing the normalized luminosity functions for galaxies with different values of $q$, we measure how the high-luminosity cutoff of the luminosity function depends on apparent axis ratio. In this way, we measure a dependence of dimming on apparent axis ratio well fit by the relation $\Delta M_{r}=1.27(\log q)^{2}$, rather than the $\Delta M \propto \log q$ law that is frequently assumed. When the absolute magnitudes of bright exponential galaxies are corrected to their "face-on" value, $M_{r}^{f}=M_{r}-\Delta M_{r}$, the average $u-r$ color is linearly dependent on $M_{r}^{f}$ for a given value of $q$; the value of $d(u-r) / d M_{r}^{f}$ ranges from $\sim 0.10$ for nearly face-on galaxies $(q>0.9)$ to $\sim 0.26$ for nearly edge-on galaxies $(q<0.3)$. When the dimming law $\Delta M_{r} \propto(\log q)^{2}$ is used to create an inclination-corrected sample of bright exponential galaxies, their apparent shapes are consistent with a distribution of mildly noncircular disks, with median short-to-long axis ratio $\gamma \approx 0.22$ and median disk ellipticity $\epsilon \approx 0.08$.
\end{abstract}

Subject headings: galaxies: fundamental parameters — galaxies: photometry — galaxies: spiral — galaxies: statistics

\section{INTRODUCTION}

Luminous galaxies ( $M_{r}<-18$ or so) can be divided into two classes, conventionally labeled "early-type" and "late-type." Early-type galaxies have redder stellar populations and a scarcity of interstellar gas and dust. The majority of luminous earlytype galaxies are elliptical galaxies, characterized by smooth isophotes and concentrated light profiles, well described by a de Vaucouleurs (1948) profile: $\log I \propto-r^{1 / 4}$. Highly luminous elliptical galaxies tend to be mildly triaxial ellipsoids (as opposed to perfectly oblate spheroids); their intrinsic short-to-long axis ratio is typically $c / a \sim 0.7$ (Ryden 1992; Vincent \& Ryden 2005). Late-type galaxies have blue stellar populations and relatively large amounts of interstellar gas and dust. The majority of luminous late-type galaxies are spiral galaxies, characterized by spiral structure within flattened disks. The disk light profile is generally well described by an exponential profile: $\log I \propto-r$. Luminous spiral galaxies are mildly elliptical (as opposed to perfectly circular) when seen face-on; their intrinsic short-to-long axis ratio is colordependent, but at visible wavelengths is typically $c / a \sim 0.25$ (Binney \& de Vaucouleurs 1981; Grosbøl 1985; Lambas et al. 1992; Fasano et al. 1993; Ryden 2004, 2006).

In a color-magnitude (CM) diagram, if the color index is chosen correctly, the early-type and late-type galaxies manifest themselves as a "red sequence" and a "blue sequence," respectively. In the Sloan Digital Sky Survey (SDSS), the distribution of $u-r$ colors for low-redshift galaxies is bimodal; Strateva (2001) finds an optimal color separator of $u-r=2.22$, when color alone is used as a discriminator between early-type and late-type galaxies. Using a full CM diagram, the color separator is found to be dependent on $M_{r}$ (Baldry et al. 2004), with the

\footnotetext{
${ }^{1}$ Center for Cosmology and Astroparticle Physics, The Ohio State University, Columbus, OH 43210.
}

color separator ranging from $u-r \approx 2.3$ for galaxies with $M_{r}<$ -21 to $u-r \approx 1.8$ for galaxies with $M_{r}>-18$.

However, a clean separation between early-type and late-type galaxies using color and absolute magnitude information alone is impossible; the red sequence and blue sequence overlap in a $\mathrm{CM}$ diagram. This overlap results partly from the fact that the color and apparent magnitude of spiral galaxies are inclinationdependent. As noted by Alam \& Ryden (2002), a sample chosen solely by the color criterion $u-r \geq 2.22$ will contain dustreddened edge-on spirals, as well as intrinsically red ellipticals.

The overlap between the red sequence and blue sequence would be reduced if we could perform an inclination correction on the colors and apparent magnitudes of spiral galaxies; that is, if we could convert observed apparent magnitudes into what they would be if the spiral galaxy were face-on. Since pivoting galaxies so that we can see them face-on is an impracticable task, we take a statistical approach to finding the average dimming, $\Delta M_{r}$, and reddening, $\Delta(u-r)$, of a spiral galaxy as a function of its inclination $i$.

In addition to allowing a cleaner separation between early-type and late-type galaxies in the CM diagram, a statistical correction for dimming and reddening has other practical uses. For instance, a flux-limited survey will undersample edge-on spirals with respect to face-on spirals; a face-on galaxy that is just above the flux limit would fall below the limit if it were edge on. With a knowledge of $\Delta M_{r}$ as a function of inclination, it is possible to create an inclination-corrected flux-limited sample, by retaining only those galaxies that would still be above the flux limit if they were tilted to be seen edge on. Since the standard technique for finding the distribution of intrinsic axis ratios of galaxies assumes a random distribution of inclinations (see, for instance, Vincent \& Ryden 2005 and references therein), such an inclination-corrected sample is essential for determining the true distribution of disk flattening. Without the inclination correction, the scarcity of 
edge-on galaxies leads to an overestimate of the typical disk thickness.

In $\S 2$ we describe how we select a sample of SDSS galaxies with exponential profiles; this gives us a population of disk-dominated spiral galaxies. The apparent axis ratio $q$ of the 25 mag $\operatorname{arcsec}^{-2}$ isophote is chosen as our surrogate for the inclination of a galaxy. In $\S 3$ we examine the luminosity function of the SDSS exponential galaxies as a function of $q$. By shifting the normalized luminosity functions until their high-luminosity cutoffs align, we can estimate the dimming $\Delta M_{r}$ of the cutoff as a function of $q$. By brightening each galaxy by the $\Delta M_{r}$ appropriate to its observed value of $q$, we find its approximate "face-on" absolute magnitude $M_{r}^{f}$. We then provide linear fits for the mean $u-r$ color as a function of $M_{r}^{f}$ for different ranges of $q$. In $\S 4$ we create an inclination-corrected flux-limited sample, which we then use to find the distribution of intrinsic short-to-long axis ratios of the SDSS exponential galaxies, confirming that they are, in fact, disks. Finally, in $\S 5$ we provide a brief discussion of what the form of $\Delta M_{r}$ as a function of $q$, and of $\Delta(u-r)$ as a function of $q$ and $M_{r}^{f}$, implies for the properties of spiral galaxies and the dust they contain.

\section{DATA}

The Sloan Digital Sky Survey (SDSS) has imaged roughly $\pi$ steradians of the sky (York et al. 2000; Adelman-McCarthy et al. 2008). SDSS photometric data is provided in five bands (ugriz) from the near-ultraviolet to the near-infrared (Fukugita et al. 1995; Smith et al. 2002). The SDSS Data Release 6 (DR6) includes $9583 \mathrm{deg}^{2}$ of photometric coverage and $7425 \mathrm{deg}^{2}$ of spectroscopic coverage (Adelman-McCarthy et al. 2008). The SDSS photometric data processing pipeline performs a morphological star/galaxy separation, with extended objects being labeled "galaxies" and pointlike objects being labeled "stars." For each galaxy, in each photometric band, two models are fitted to the galaxy image. One model has a de Vaucouleurs profile (de Vaucouleurs 1948):

$$
I(R)=I_{e} \exp \left\{-7.67\left[\left(R / R_{e}\right)^{-1 / 4}-1\right]\right\},
$$

truncated beyond $7 R_{e}$ to go smoothly to zero at $8 R_{e}$. The other model has an exponential profile:

$$
I(R)=I_{e} \exp \left[-1.68\left(R / R_{e}-1\right)\right]
$$

truncated beyond $3 R_{e}$ to go smoothly to zero at $4 R_{e}$. The SDSS DR6 pipeline also takes the best-fitting de Vaucouleurs model and exponential model for each galaxy, and finds the linear combination of the two that best fits the galaxy image. The fraction of the total flux contributed by the de Vaucouleurs component is the parameter fracDeV, which is constrained to lie in the interval $0 \leq$ fracDeV $\leq 1$. The fracDeV parameter thus acts as a concentration index, varying from fracDeV $=1$ for highly concentrated de Vaucouleurs galaxies to fracDeV $=0$ for less concentrated exponential galaxies.

As our measure of the flux of each galaxy, we use the $r$-band "model magnitude"; for galaxies with fracDeV $\geq 0.5$, the model magnitude is the integrated magnitude of the de Vaucouleurs model, and for galaxies with fracDeV $<0.5$ it is the integrated magnitude of the exponential model. As our measure of the color of each galaxy, we use the $u-r$ color, defined as the difference between the $u$-band model magnitude and the $r$-band model magnitude. As our measure of the axis ratio of each galaxy, we use the axis ratio of the $r$-band $25 \mathrm{mag} \operatorname{arcsec}^{-2}$ isophote. The

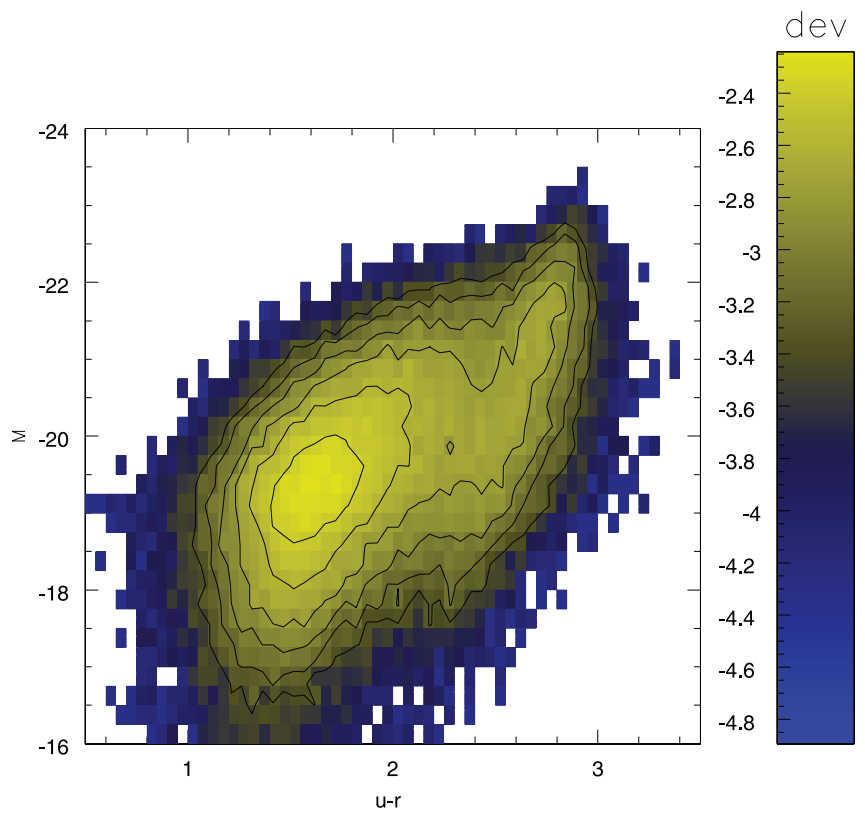

FIG. 1.-Color-magnitude diagram (absolute $r$-band magnitude vs. $u-r$ ) for all galaxies in our flux-limited $z<0.06$ sample. The color bar on the right indicates the value of $\log _{10} F$ corresponding to each color, where $F$ is the fraction of the galaxies in each bin. Bin sizes are $\Delta M_{r}=0.25$ and $\Delta(u-r)=0.05$.

SDSS DR6 data pipeline finds the best-fitting ellipse to the $25 \mathrm{mag} \operatorname{arcsec}^{-2}$ isophote in each band; the semimajor and semiminor axes of this isophotal ellipse are $A_{25}$ and $B_{25}$, respectively. The isophotal axis ratio $q_{25} \equiv B_{25} / A_{25}$ then provides a measure of the apparent galaxy shape at a few times the effective radius; for galaxies with fracDeV $=0$, the average value of $A_{25} / R_{e}$ is about 2.4 (Vincent \& Ryden 2005).

Our full sample of galaxies consists of objects in the SDSS DR6 spectroscopic sample that are labeled as galaxies and that have spectroscopic redshifts $z>0.004$, to eliminate contaminating foreground objects, and $z<0.06$, to reduce the possibility of weak-lensing distortions of apparent shapes, and to eliminate, in practice, the necessity of applying $K$-corrections. To eliminate low-quality redshifts, we require that the SDSS redshift confidence parameter have a value $z$ Conf $>0.35$. To ensure that the galaxies in our full sample are well resolved spatially, we require that their photometric data fulfill the criterion $\tau>6.25 \tau_{\mathrm{psf}}$, where $\tau$ is the adaptive second-order moment of the galaxy image and $\tau_{\text {psf }}$ is the adaptive second-order moment of the point-spread function at the galaxy's location. Our full galaxy sample, defined in this way, contains $n=78,230$ galaxies.

Figure 1 shows the color-magnitude diagram for the 78,230 galaxies in our full sample. The absolute magnitude $M_{r}$ is computed from the model magnitude $m_{r}$ assuming a Hubble constant $H_{0}=70 \mathrm{~km} \mathrm{~s}^{-1} \mathrm{Mpc}^{-1}$ in a flat universe with mass contributing $\Omega_{m, 0}=0.3$ to the density parameter and a cosmological constant contributing $\Omega_{\Lambda, 0}=0.7$. No $K$-corrections are applied. In Figure 1, the division between the blue sequence, on the left, and the red sequence, on the right, can be clearly seen. However, the so-called "green valley," between the blue and red sequences, is well populated with galaxies.

Using the information about surface brightness profiles provided by the fracDeV parameter, we can isolate subsamples that are all late-type or all early-type galaxies. Of our full sample, $n=36,162$ galaxies have fracDeV $\leq 0.1$. The $\mathrm{CM}$ diagram for this exponential subsample is shown in Figure 2 (left). The exponential galaxies fall along a well-defined blue sequence. Note, 

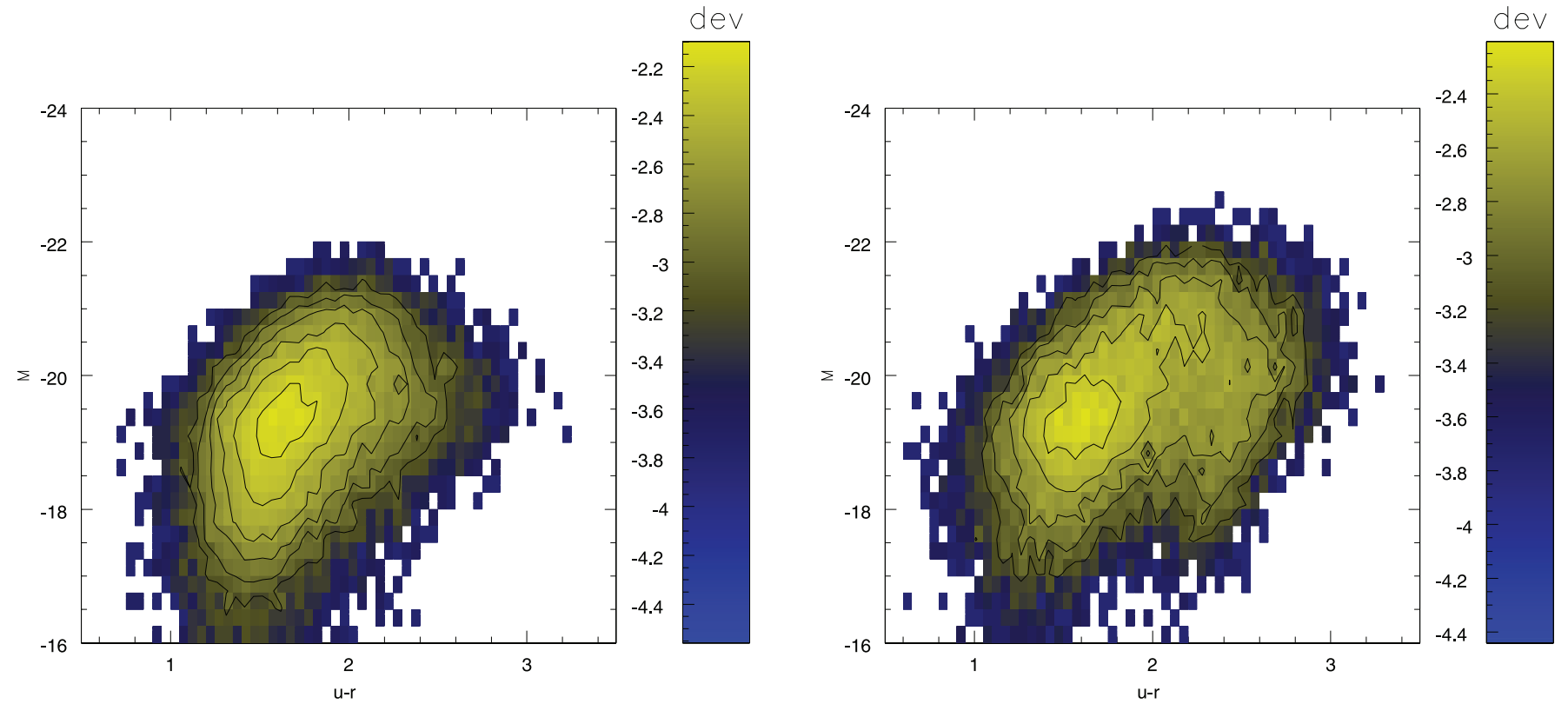

FIG. 2. Left: Same as Fig. 1, but containing only those galaxies with fracDeV $\leq 0.1$. Right: Same as Fig. 1, but containing only those galaxies with $0.1<$ fracDeV $\leq 0.5$.

however, that the spread in $u-r$ color increases with increasing luminosity. For comparison, Figure 2 (right) shows the CM diagram for the $n=27,567$ galaxies in our full sample that have $0.1<$ fracDeV $\leq 0.5$. These more concentrated galaxies fall primarily along the blue sequence; however, there are a significant number at the faint end of the the red sequence $\left(M_{r} \sim-19.5\right.$, $u-r \sim 2.4$ ). Moving to greater concentration, Figure 3 (left) shows the CM diagram for the $n=11,202$ galaxies that have $0.5<$ fracDeV $\leq 0.9$. These galaxies fall primarily along the red sequence; however, there are a significant number at the bright end of the blue sequence $\left(M_{r} \sim-20, u-r \sim 1.6\right)$. Finally, Figure 3 (right) shows the CM diagram for the relatively small number of galaxies in the full sample $(n=3299)$ that have fracDeV $>0.9$. These de Vaucouleurs galaxies represent the high-luminosity end of the red sequence.

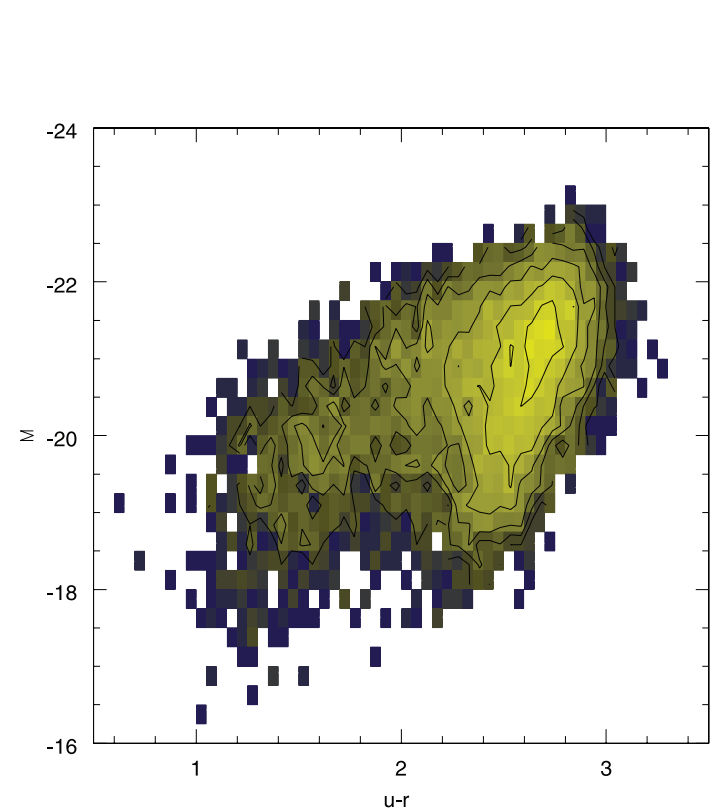

Previous studies of the colors, absolute magnitudes, and apparent shapes of late-type galaxies in the Sloan Digital Sky Survey have used different definitions of "late-type galaxy." Blanton et al. (2003), for instance, find that galaxies with Sérsic (1968) index $n<1.5$ fall on the blue sequence of a CM diagram, while those with Sérsic index $n>3$ fall on the red sequence. (A galaxy with a perfect Sérsic profile will have fracDeV $\approx 0.3$ if $n=1.5$ and fracDeV $\approx 0.8$ if $n=3$.) Chang et al. (2006) and Shao et al. (2007), in their studies of late-type galaxies in the SDSS, find it useful to chose a sample with fracDeV $<0.5$. This cut in fracDeV, however, does allow some galaxies from the red sequence to enter the sample. Since the fracDeV distribution is strongly peaked at fracDeV $=0$, we choose to make the more stringent cut fracDeV $\leq 0.1$ to create our late-type galaxy subsample. The $n=36,162$ galaxies with fracDeV $\leq 0.1$, we
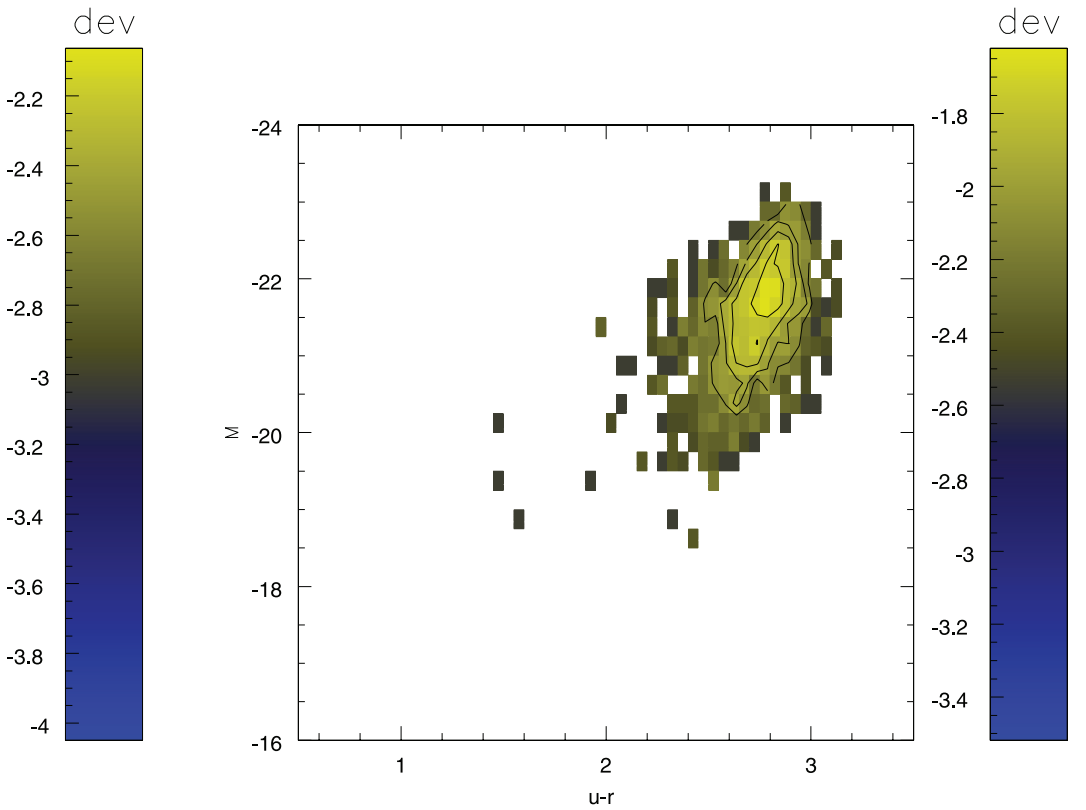

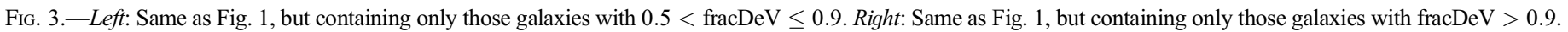




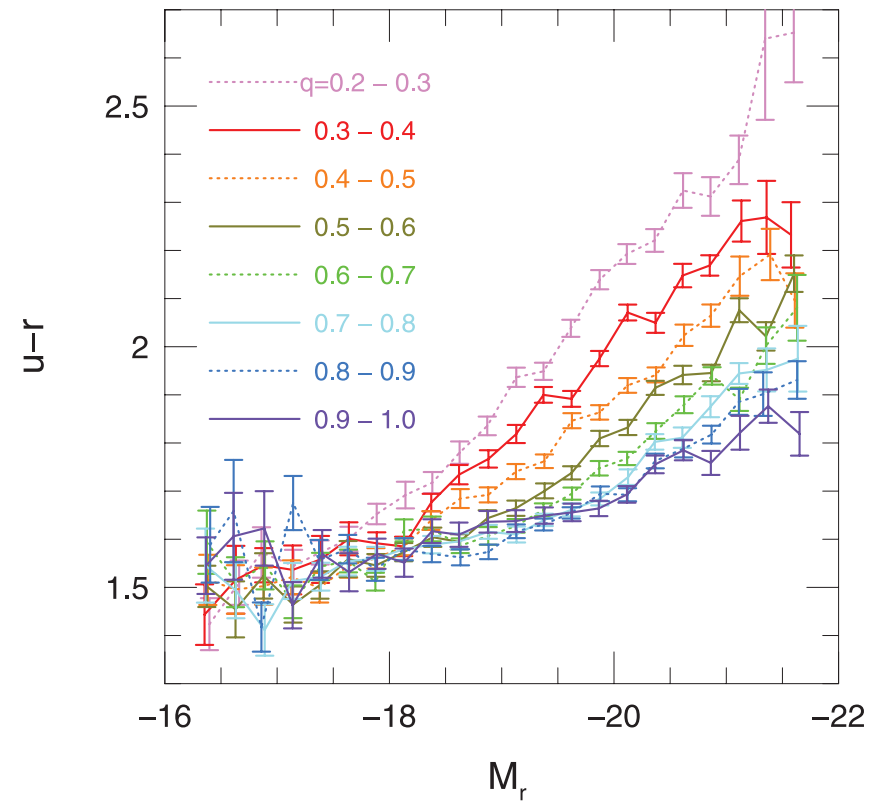

FIG. 4.-Average $u-r$ color as a function of $M_{r}$ for our flux-limited, $z<0.06$ sample of exponential galaxies. Results are shown for different ranges of $q$, the apparent axis ratio. Error bars represent the estimated error in the mean color.

expect, will provide us with a sample of late-type spirals whose light is dominated by a dusty exponential disk.

\section{ANALYSIS}

How do we know that our subsample of (nearly) exponential galaxies are actually flattened disks? We have, after all, chosen them solely on the basis of their surface brightness profiles. Although the disks of spiral galaxies are known to be well fitted by exponential profiles, so are other subclasses of galaxies, such as dwarf ellipticals. We can test the assertion that exponential galaxies are flattened, dust-containing disks by looking at their colors as a function of apparent axis ratio (if a galaxy is a disk, then its apparent axis ratio $q$ will be smallest when it is edge-on). Figure 4 is a plot of the mean $u-r$ color as a function of absolute magnitude $M_{r}$ for the $n=36,162$ galaxies in our exponential subsample (fracDeV $\leq 0.1$ ). The galaxies are binned by their apparent axis ratio $q$. Note that for exponential galaxies brighter than $M_{r} \sim-19$, there is a noticeable correlation between $q$ and $u-r$ at a given absolute magnitude, with smaller values of $q$ corresponding to larger values of $u-r$. This is just what we expect for a population of dusty disk galaxies. However, at $M_{r}>$ -18 , there is no correlation between $q$ and $u-r$. At these low luminosities, the galaxies in the exponential subsample are blue $(u-r \sim 1.5)$ dwarf galaxies in which the stars and dust are not in orderly thin disks. Thus, in looking for inclination-dependent colors and magnitudes, we look at those high-luminosity $\left(M_{r} \lesssim\right.$ $-19)$ exponential galaxies for which the color actually is inclination-dependent.

We can further test our assertion that luminous exponential galaxies are disks by looking at their distribution of apparent axis ratios. Figure 5 shows the distribution of $q$ for the galaxies in our exponential subsample that have $M_{r} \leq-19.4$. If the galaxies were infinitesimally thin, perfectly circular disks, with randomly chosen viewing angles, then the distribution of $q$ would be flat. Although the distribution shown in Figure 5 is flat from $q \sim 0.35$ to $q \sim 0.9$, there is a scarcity of galaxies with smaller and larger values of $q$. The lack of galaxies with $q>0.9$ results from the fact that disk galaxies are not perfect circles (Ryden

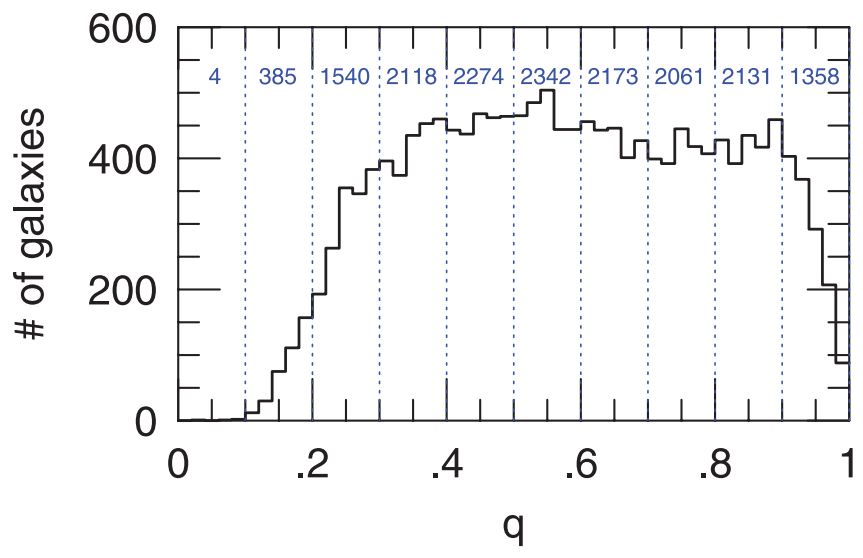

FIG. 5.-Distribution of apparent axis ratio $q$ for the galaxies in our fluxlimited, $z<0.06$ sample of exponential galaxies that have $M_{r}<-19.4$. The numbers given between each pair of dotted lines is the total number of galaxies in that $\Delta q=0.1$ range.

2004). The lack of galaxies with $q<0.2$ results from the fact that disk galaxies are not infinitesimally thin. However, there is another reason for the lack of galaxies in the range $q<0.35$; the disk galaxies contributing to the histogram of Figure 5 are not randomly oriented. Since the SDSS spectroscopic survey is flux-limited, dimmed edge-on galaxies are undercounted relative to minimally dimmed face-on galaxies.

Visual inspection of SDSS "postage stamp" images reveals that high-luminosity exponential galaxies at low redshift $\left(M_{r}<\right.$ -19.4 , fracDeV $\leq 0.1$, and $z<0.06$ ) typically have the appearance of disk-dominated galaxies with small bulges, consistent with a Hubble type of Sbc or Sc. Our visual impressions are consistent with the analysis of Ball et al. (2006) who assigned Hubble types to SDSS galaxies with the assistance of an artificial neural network (Ball et al. 2004). Galaxies in the blue sequence with $-18.3<M_{r}<-20.3$ were found to have a most probable Sérsic index $n \approx 1.2$, corresponding to fracDeV $\approx 0.1$; the most probable Hubble $T$ type assigned to the same galaxies was $T \approx$ 3.5 , corresponding to an Sbc galaxy. Thus, our selection criteria exclude early-type (Sa) spirals by requiring fracDeV $\leq 0.1$ and exclude late-type (Sd) spirals by requiring $M_{r}<-19$.

In general, when a spiral galaxy is seen at an arbitrary inclination, it will be fainter at visible wavelengths than it would be seen face-on. We may write

$$
M_{r}=M_{r}^{f}+\Delta M_{r}
$$

where $M_{r}$ is the $r$-band absolute magnitude computed from the actual apparent magnitude and redshift, $M_{r}^{f}$ is what the absolute magnitude would be if the galaxy were seen face-on, and $\Delta M_{r}$ is the inclination-related dimming. In general, $\Delta M_{r}$ will be a function of the (unknown) inclination $i$ as well as of the detailed properties of the observed galaxy's dust. We assume that the apparent axis ratio $q$ of the $25 \mathrm{mag} \operatorname{arcsec}^{-2}$ isophote is an adequate measure of the inclination. If every spiral galaxy were a perfect oblate spheroid, with intrinsic short-to-long axis ratio $\gamma=c / a$, then the inclination $i$ would be uniquely determined by the apparent axis ratio $q$, through the usual relation

$$
\cos ^{2} i=\left(q^{2}-\gamma^{2}\right) /\left(1-\gamma^{2}\right)
$$

One source of error in this relation is that spiral galaxies do not all have the same intrinsic thickness $\gamma$. However, an erroneous assumed value for $\gamma$ is irrelevant in the case of low inclination 
and high $q$ : a face-on thick disk has the same apparent shape as a face-on thin disk. An erroneous assumed value for $\gamma$ produces the largest error in $i$ when the apparent axis ratio $q$ is small: a circular disk with $q=0.3$ is at an inclination $\cos i=0$ if it is a fat disk with $\gamma=0.3$, but at an inclination $\cos i=0.28$ if it is an ultrathin disk with $\gamma=0.1$. Another source of error in equation (4) is that disks are not perfectly circular. Consider an ultrathin disk with $\epsilon \approx 0.1$, a typical ellipticity for a spiral galaxy. When the galaxy is face-on, equation (4) will yield $\cos i \approx 0.9$, instead of the true value of $\cos i=1$. When the same disk is viewed at a high inclination, the computed value of $\cos i$ has an error $\sim \epsilon \cos 2 \phi$, where $\phi$ is the azimuthal viewing angle measured relative to the intrinsic long axis of the disk (Ryden 2006). Thus, when we use $q$ as a surrogate for the inclination $i$, we should remember that two spiral galaxies with the same $q$ may have $\cos i$ differing by as much as $\sim \epsilon$, where $\epsilon$ is their average intrinsic disk ellipticity.

Finding $\Delta M$ as a function of $q$ is a nontrivial task. The pioneering analysis of Holmberg (1958) looked at the "surface magnitude" $S_{0}=m+5 \log a$, where $m$ was the integrated photographic magnitude of a spiral galaxy and $a$ was the major axis of the $m_{\mathrm{pg}}=26.5$ isophote. Holmberg (1958) found, for spirals of any given Hubble type, a dependence of $S_{0}$ on $q$, with lower values of $q$ corresponding to larger values of $S_{0}$. However, the interpretation of Holmberg's results is subject to ambiguity (Valentijn 1990). For a perfectly transparent disk, $m$ is independent of $q$, but the isophotal radius $a$ increases as $q$ decreases. Conversely, for a totally opaque disk, $a$ is independent of $q$, but $m$ increases as $q$ decreases. To avoid the ambiguities of Holmberg's method, we will use a different approach.

Our statistical method for finding $\Delta M_{r}$ as a function of $q$ involves comparing the luminosity function for exponential galaxies with different values of $q$. We begin by creating a volume-limited subsample of our exponential (fracDeV $\leq 0.1$ ) SDSS galaxies. The redshift limit of the volume-limited subsample is $z=0.06$; the flux limit is $m_{r}=17.77$, resulting in a low-luminosity cutoff at $M_{r}=-19.4$. (The SDSS spectroscopic survey is complete to a limiting Petrosian apparent magnitude $r=17.77$. For galaxies that are well fitted by the exponential model, the Petrosian apparent magnitudes and the model magnitudes in our analysis are very similar, with an rms scatter of $<0.1 \mathrm{mag}$.) We then take the $n=16,363$ exponential galaxies in the volume-limited subsample and bin them by apparent axis ratio, in bins of width $\Delta q=0.1$. The galaxies with $0.9<q \leq 1.0$ are galaxies that we expect to be nearly face-on.

The cumulative luminosity function for the exponential galaxies with $0.9<q \leq 1.0$ is shown as the solid line in Figure 6 (top). Since we expect $\Delta M_{r}=0$ for these nearly face-on galaxies, this solid line is our estimator of the normalized luminosity function $f\left(M_{r}^{f}\right)$ for face-on spiral galaxies brighter than $M_{r}^{f}=-19.4$. The observed luminosity function for these bright, nearly face-on spirals is well fit by a Schechter (1976) function:

$$
\Phi\left(M_{r}\right) \propto 10^{0.4(\alpha+1)\left(M_{r}^{*}-M_{r}\right)} \exp \left[-10^{0.4\left(M_{r}^{*}-M_{r}\right)}\right] .
$$

The best-fitting Schechter function for the $q>0.9$ exponential galaxies, shown as the open circles in Figure 6 , has $\alpha=-0.69$ and $M_{r}^{*}=-19.96$; a Kolmogorov-Smirnov (KS) test yields a probability $P_{\mathrm{KS}}=0.97$ for the observed distribution compared to the Schechter function. Thus, there is a distinct feature in the luminosity function for $q>0.9$ galaxies - an exponential cutoff at luminosities greater than $M_{r} \sim-20$.

For comparison, the dashed line in Figure 6 (top) shows the cumulative luminosity function for exponential galaxies with

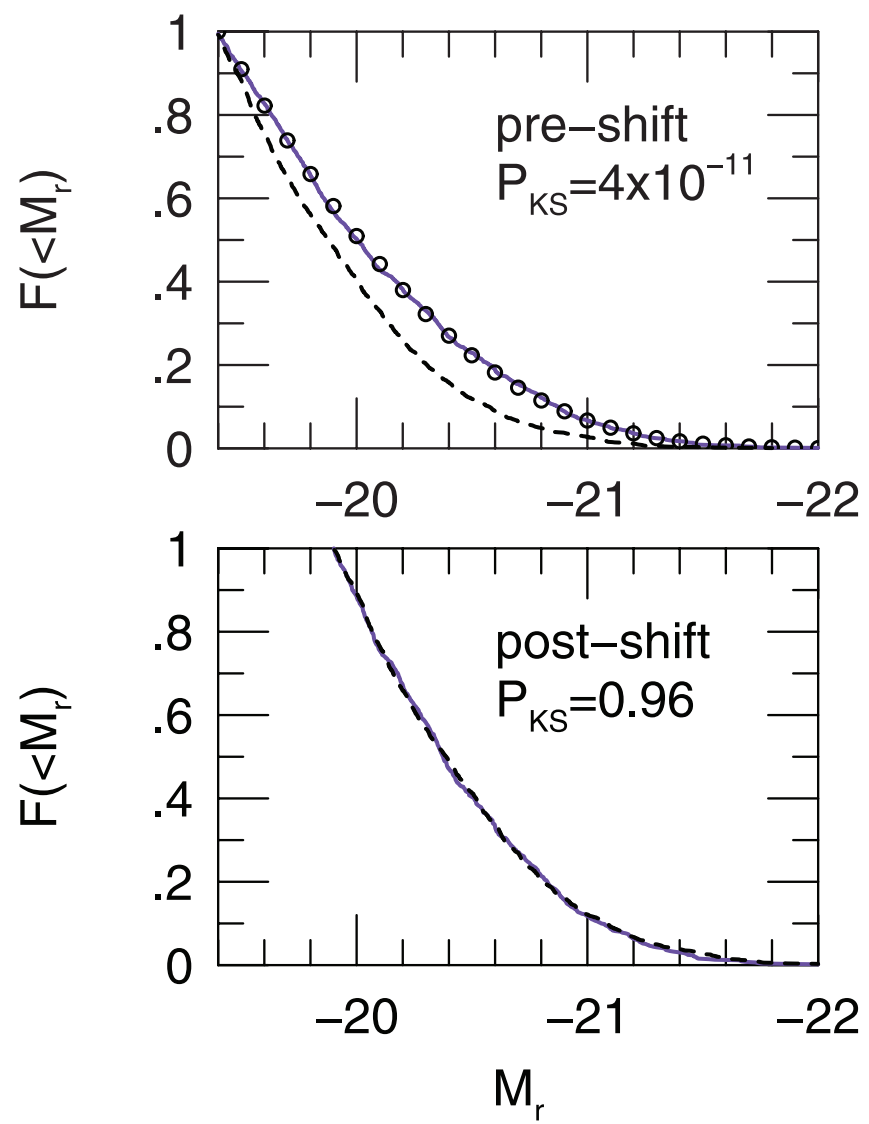

FIG. 6.-Top: Cumulative luminosity function for the galaxies in the volumelimited sample with $q>0.9$ (solid line) and with $0.2<q \leq 0.3$ (dotted line). Functions are normalized so that $F\left(<M_{r}\right)=1$ at $M_{r}=-19.40$. Open circles represent the best-fitting Schechter function $\left(\alpha=-0.69, M_{r, *}=-19.97\right)$ for the galaxies with $q>0.9$. Bottom: Comparison of the cumulative luminosity functions after the $0.2<q \leq 0.3$ subsample has been shifted by $-\Delta M_{r}=-0.51$. Functions are normalized so that $F\left(<M_{r}\right)=1$ at $M_{r}=-19.40-0.51$.

$0.2<q \leq 0.3$; we expect these galaxies to be nearly edge-on. The luminosity function for the $q \sim 0.25$ galaxies is different from that of the $q>0.9$ galaxies; a KS test yields a probability $P_{\mathrm{KS}}=4 \times 10^{-11}$. However, if we take each galaxy in the $q \sim 0.25$ bin and brighten by the same amount $\Delta M_{r}$, we can compare this artificially brightened luminosity function to the luminosity function for face-on $(q>0.9)$ galaxies brighter than $M_{r}=-19.4-\Delta M_{r}$. The comparison between the two cumulative luminosity functions is done using a KS test. Figure 6 (bottom) shows the comparison between the $0.2<q \leq 0.3$ galaxies and the $q>0.9$ galaxies, using the optimal shift $\Delta M_{r}=0.51$. The KS test yields a probability $P_{\mathrm{KS}}=0.96$ for this shift. Note that our method for finding $\Delta M_{r}(q)$ is essentially asking where the high-luminosity cutoff is for each luminosity function; the luminosity function for the galaxies with the lower value of $q$ is then shifted until its cutoff aligns with the cutoff for the $q>0.9$ luminosity function. As a result, the value of $\Delta M_{r}$ that we measure is the dimming appropriate for a galaxy whose face-on absolute magnitude is $M_{r}^{f} \approx M_{r}^{*} \approx-20$.

Figure 7 gives a summary of the best-fitting absolute magnitude shift $\Delta M_{r}$ as a function of $q$ for the exponential galaxies. Each data point gives the value of $\Delta M_{r}$ which maximizes the KS probability when the luminosity function of galaxies in a particular range of $q$ are compared to galaxies with $q>0.9$. The error bars indicate the range of $\Delta M_{r}$ over which $P_{\mathrm{KS}}>0.1$. Because the high-luminosity cutoff in the luminosity function is 


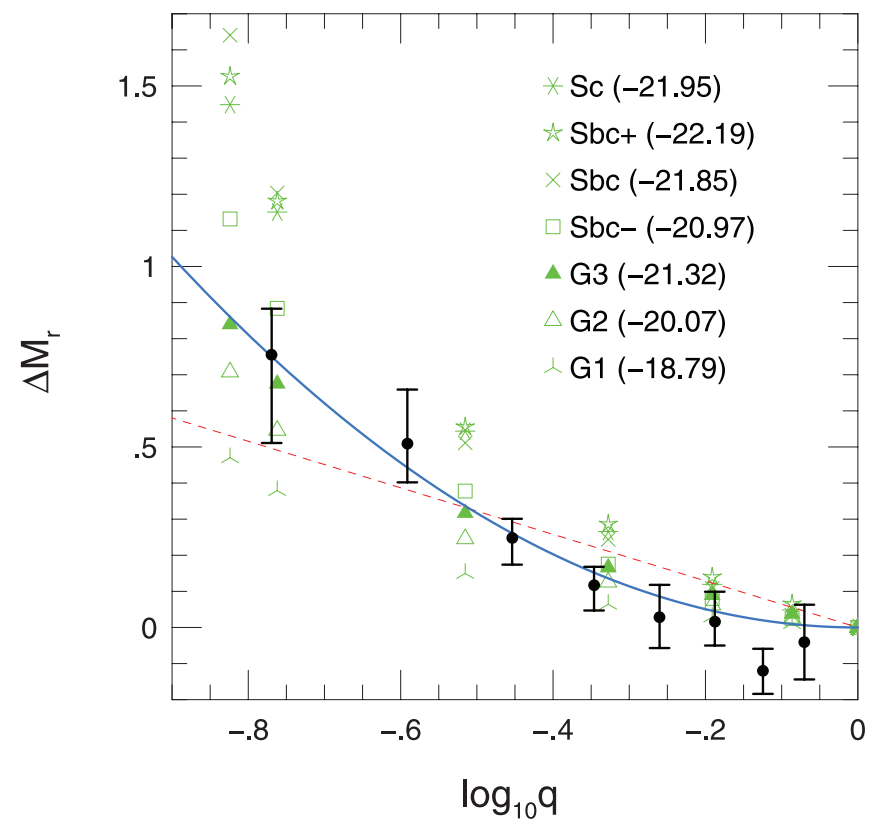

FIG. 7.-Points show the best-fitting shift $\Delta M_{r}$ of the exponential galaxy luminosity function as a function of the apparent axis ratio $q$. The error bars indicate the range of $\Delta M_{r}$ for which $P_{\mathrm{KS}}>0.1$. The dashed red curve shows $\Delta M_{r}=$ $-0.64 \log q$, and the solid blue curve shows $\Delta M_{r}=1.27(\log q)^{2}$. Green symbols show the results for the numerically modeled galaxies of (Rocha et al. 2008), as discussed in $\S 5$.

not a perfectly sharp feature, the range of statistically acceptable values of $\Delta M_{r}$ is as large as $\sim 0.4$ mag in the low- $q$ bins, where there are relatively few galaxies. A common parameterization of the $\Delta M-q$ relation, following the Third Reference Catalogue (de Vaucouleurs et al. 1991), is

$$
\Delta M=-\gamma_{\lambda} \log q
$$

where the value of $\gamma_{\lambda}$ depends on the wavelength of observation, and on the population of galaxies observed. de Vaucouleurs et al. (1991) used $\gamma_{B}=1.5$ for spiral galaxies of type Sc observed in the $B$ band. For pure disk systems (type $\mathrm{Sc}-\mathrm{Sd}$ ), Bottinelli et al. (1995) found $\gamma_{B}=1.67$. Tully et al. (1998) found dimming that was dependent on luminosity as well as on wavelength: for bright spirals in the Ursa Major and Pisces clusters, they found $\gamma_{B}=1.7, \gamma_{R}=1.3$, and $\gamma_{I}=1.0$ in the $B, R$, and $I$ bands. Shao et al. (2007), looking at fracDeV $<0.5$ galaxies in the SDSS, found values of $\gamma$ ranging from $\gamma_{u}=2.19$ in the $u$ band, through $\gamma_{r}=1.37$ in the $r$ band, to $\gamma_{z}=0.80$ in the $z$ band.

Our best fit to the logarithmic relation of equation (6) is shown as the dashed line in Figure 7; the best-fitting value of $\gamma$ is $\gamma_{r}=0.64$. However, the $\Delta M_{r} \propto \log q$ fit overestimates the dimming of galaxies with $q>0.4$, and underestimates the dimming of nearly edge-on galaxies with $q<0.3$. In fact, our data are consistent with no dimming at all in the $r$ band for galaxies with $q>0.5$. A superior fit is provided by the solid line in Figure 7, which represents a dimming proportional to the square of $\log q$ :

$$
\Delta M_{r}=\beta_{r}(\log q)^{2},
$$

with $\beta_{r}=1.27$. Our results are in qualitative agreement with those of Masters et al. (2003) who find that for galaxies in the Two Micron All-Sky Survey Extended Source Catalog, the simple linear extinction law of equation (6) gives an inadequate fit to the dimming in near-infrared $\left(J, H\right.$, and $\left.K_{S}\right)$ bands. A better fit is

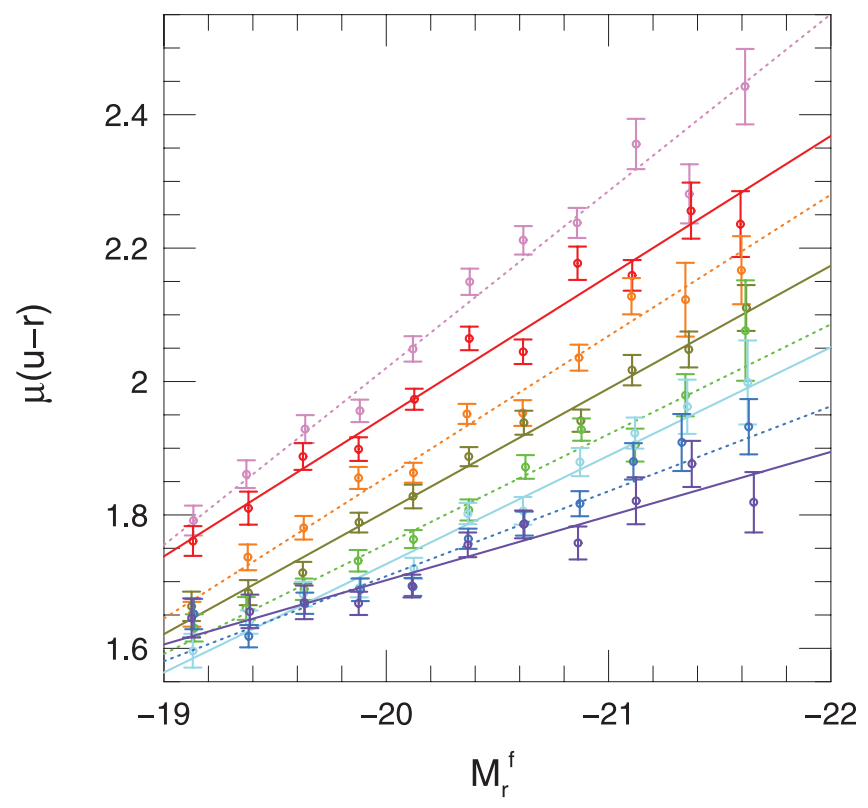

FIG. 8.-Average $u-r$ color as a function of the corrected, "face-on" absolute magnitude $M_{r}^{f}$ for our flux-limited, $z<0.06$ sample of exponential galaxies. Colors and line types are the same as in Fig. 4. Error bars represent the estimated error in the mean color.

provided by a quadratic in $\log q$. In the $J$ band, for instance, Masters et al. (2003) find the best quadratic fit is $\Delta M_{J}=$ $0.12 \log q+1.14(\log q)^{2}$. If we attempt a fit of this form to our exponential $r$-band subsample, we find $\Delta M_{r}=0.25 \log q+$ $1.66(\log q)^{2}$. However, this does not provide a statistically better fit, given the loss of a degree of freedom, than the simpler parabolic form of equation (7).

Given the dimming correction of equation (7), we can compute the "face-on" absolute magnitude $M_{r}^{f}$ for every exponential galaxy with measured $m_{r}, z$, and $q$ :

$$
M_{r}^{f}=M_{r}-\Delta M_{r}=M_{r}-1.27(\log q)^{2} .
$$

Figure 8 shows the average $u-r$ color for exponential galaxies, as a function of the corrected absolute magnitude $M_{r}^{f}$. We look only at galaxies with $M_{r}^{f}<-19$, to exclude the dwarf galaxies for which the correction is inappropriate. The different colors and line types in Figure 8 represent different values of the apparent axis ratio $q$, just as in Figure 5. For each range of $q$, the mean color, $\langle u-r\rangle$, is linear in the corrected absolute magnitude, $M_{r}^{f}$, with brighter galaxies being redder, on average. The $q>0.9$ galaxies, which are nearly face-on, have a relatively small dependence of average color on absolute magnitude; for the $q>0.9$ galaxies, increasing the brightness by $1 \mathrm{mag}$ in the $r$ band corresponds to reddening the galaxy by $\sim 0.10 \mathrm{mag}$, on average, in $u-r$. This correlation is a manifestation of the dependence of stellar population on galaxy luminosity; in general, more luminous spiral galaxies have populations that are older and more metal-rich (Bell \& de Jong 2000; MacArthur et al. 2004). The nearly edge-on galaxies $(q<0.3)$ have a stronger dependence of average color on absolute magnitude; for the $q<0.3$ galaxies, an increase in brightness by $1 \mathrm{mag}$ in the $r$ band corresponds to a reddening of $\sim 0.27 \mathrm{mag}$, on average, in $u-r$. The steepness of the mean color-luminosity relation for nearly edge-on spiral galaxies is a manifestation of the dependence of dust opacity on absolute magnitude; in general, more luminous spiral galaxies, being more metal-rich, have greater disk opacity due to dust (Masters et al. 2003). 
TABLE 1

Mean Color vs. Face-on Luminosity: $\mu(u-r)=a+b\left(M_{r}^{f}+20.5\right)$

\begin{tabular}{ccc}
\hline \hline$q$ & $a$ & $b$ \\
\hline $0.26 \ldots \ldots \ldots \ldots \ldots \ldots$. & 2.15 & -0.265 \\
$0.35 \ldots \ldots \ldots \ldots \ldots \ldots \ldots$ & 2.05 & -0.210 \\
$0.45 \ldots \ldots \ldots \ldots \ldots \ldots$. & 1.96 & -0.212 \\
$0.55 \ldots \ldots \ldots \ldots \ldots \ldots \ldots$ & 1.90 & -0.184 \\
$0.65 \ldots \ldots \ldots \ldots \ldots \ldots \ldots$. & 1.84 & -0.165 \\
$0.75 \ldots \ldots \ldots \ldots \ldots \ldots \ldots$. & 1.81 & -0.162 \\
$0.85 \ldots \ldots \ldots \ldots \ldots \ldots \ldots$ & 1.77 & -0.127 \\
$0.95 \ldots \ldots \ldots \ldots \ldots \ldots \ldots$. & 1.75 & -0.096 \\
\hline
\end{tabular}

By doing a least-squares fit to the function

$$
\langle u-r\rangle=a+b\left(M_{r}^{f}+20.5\right),
$$

we find the straight lines in Figure 8; the intercepts $(a)$ and slopes $(b)$ are given in Table 1 . The mean $u-r$ color for an exponential galaxy with $M_{r}^{f}=-20.5$ is nearly linear in $\log q$; our best fit is

$$
a=1.72-0.723 \log q .
$$

The best-fitting function of a similar form for $b$ as a function of $q$ is

$$
b=-0.11+0.255 \log q .
$$

We can compute a corrected, "face-on" color, $(u-r)^{f}$, for each galaxy using the relation

$$
(u-r)^{f}=(u-r)+\left[0.723-0.255\left(M_{r}^{f}+20.5\right)\right] \log q,
$$

where $u-r$ is the color computed from observations, and $M_{r}^{f}$ is the corrected absolute magnitude from equation (8). To illustrate the effects of using the "face-on" colors and absolute magnitudes on the CM diagram, Figure 9 shows the CM diagram for the exponential (fracDeV $<0.1$ ) galaxies in our flux-limited $z<$ 0.06 sample. The left panel uses the corrected absolute magnitudes and colors from equations (8) and (12), while the right panel uses the uncorrected values. Of the 21,813 galaxies with $M_{r}<-19$ plotted in Figure 9 (right), 5213 (23.9\%) have a $u-r$ color redder than the optimal separator of Baldry et al. (2004) intended to divide the red sequence from the blue sequence with optimal effectiveness. By contrast, of the 23,602 galaxies with $M_{r}^{f}<-19$ plotted in the left panel, only 2335 (9.9\%) have colors redder than the optimal dividing color of Baldry et al. (2004).

The color correction of equation (12) is an average correction; the actual correction for any individual galaxy will be different, due to the galaxy-to-galaxy variation in dust properties. The consequence of the variation is seen in Figure 10, which shows the standard deviation in the $u-r$ color, as a function of $M_{r}^{f}$, for different values of $q$. For nearly face-on $(q>0.9)$ exponential galaxies, the standard deviation in color is luminosity dependent, decreasing from $\sigma(u-r) \sim 0.3$ at $M_{r}^{f} \sim-19$ to $\sigma(u-r) \sim 0.2$ at $M_{r}^{f} \sim-21.5$. For nearly edge-on galaxies $(q<0.3)$, the standard deviation is greater at any given value of $M_{r}^{f}$, ranging from $\sigma(u-r) \sim 0.35$ at $M_{r}^{f} \sim-19$ to $\sigma(u-r) \sim 0.3$ at $M_{r}^{f} \sim$ -21.5 . At any value of $M_{r}^{f}$, the edge-on galaxies have an excess in $\sigma(u-r)$, caused by variations in dust properties, of $\Delta \sigma \sim 0.23$, added in quadrature.

\section{INTRINSIC SHAPES}

Our analysis has implicitly assumed that bright SDSS galaxies with fracDeV $<0.1$ are flattened, nearly circular disks. Now that we have an empirical correction for dimming as a function of apparent axis ratio (eq. [8]), we can test whether this assumption is self-consistent. If a population of galaxies consists of oblate spheroids with a random distribution of inclinations, the observed distribution of apparent axis ratios, $f(q)$, can be inverted to find the distribution of intrinsic axis ratios, $f(\gamma)$ (Hubble 1926; Sandage et al. 1970).

For a population of dusty disk galaxies whose apparent magnitude depends on inclination, selecting a subsample whose inclinations are random requires a little extra care. A simple
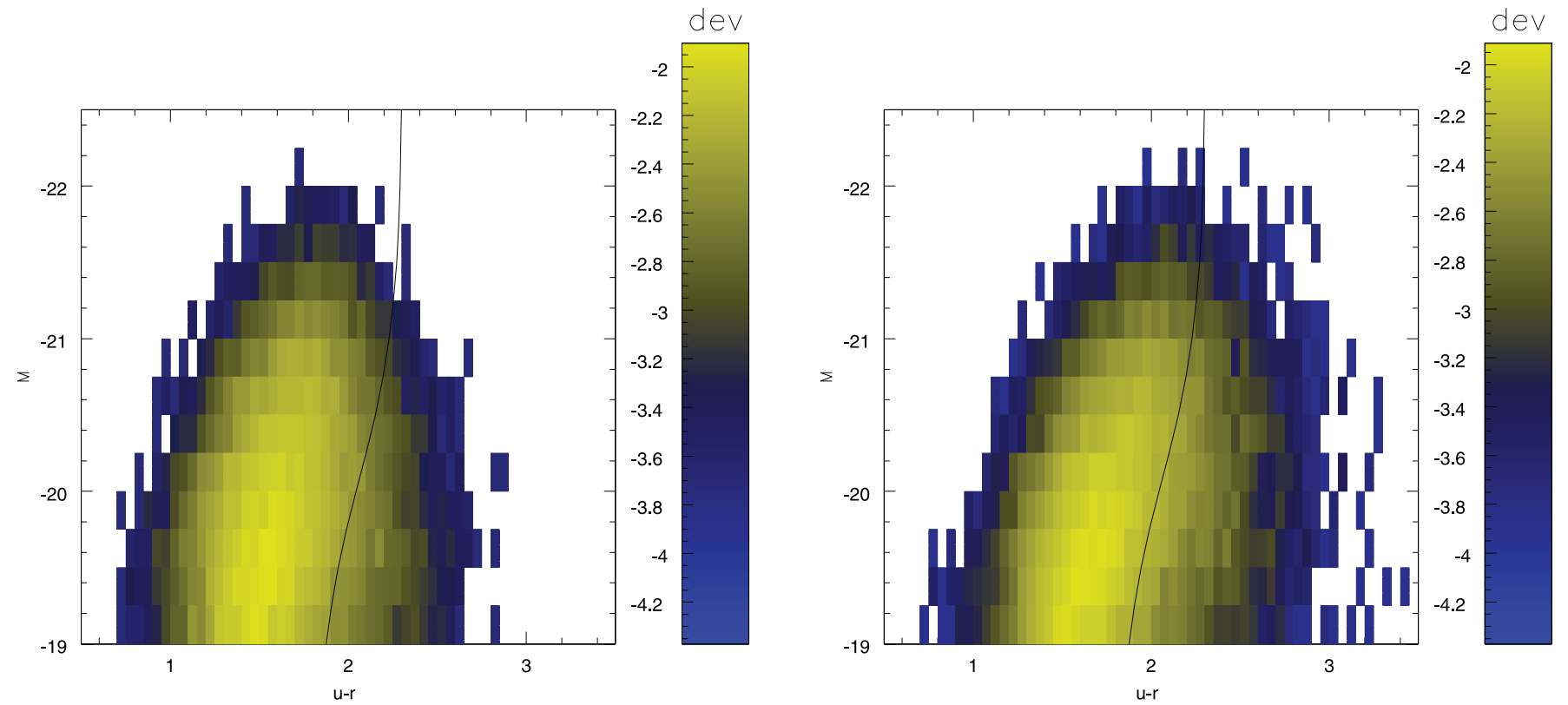

FIG. 9.-Left: Color-magnitude diagram for fracDeV $<0.1$ galaxies, using the corrected $(u-r)^{f}$ color (eq. [12]) and the corrected $M_{r}^{f}$ absolute magnitude (eq. [8]). Right: Same as left panel, but using uncorrected $u-r$ color and $M_{r}$ absolute magnitude. 


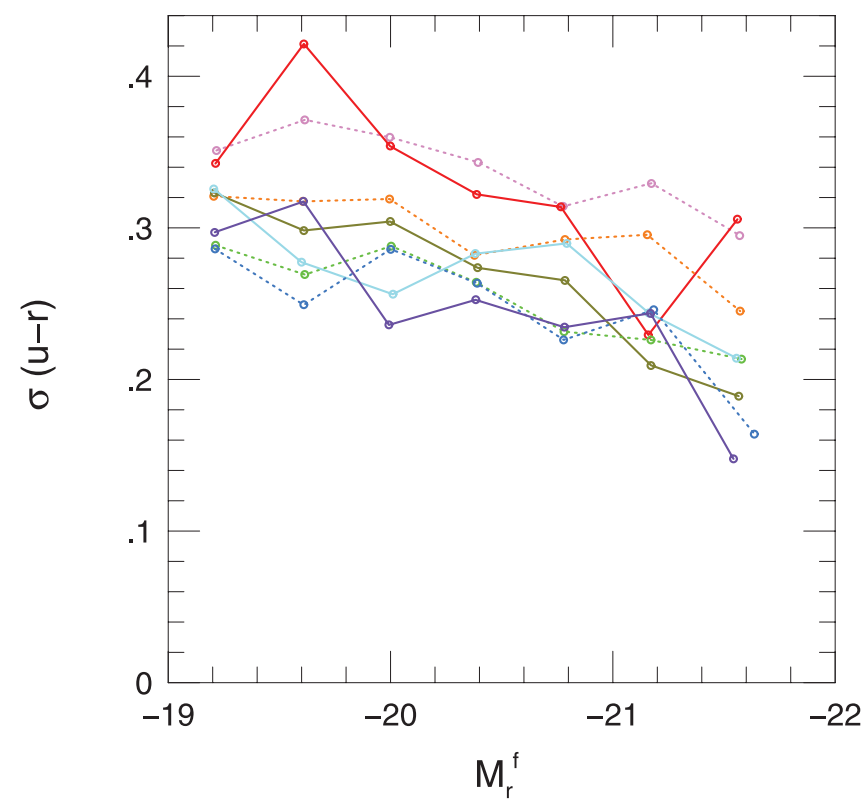

FIg. 10.-Standard deviation in the $u-r$ color as a function of the corrected absolute magnitude $M_{r}^{f}$ for our flux-limited, $z<0.06$ sample of exponential galaxies. Colors and line types are the same as in Fig. 4.

flux-limited subsample will be biased against disks at high inclination. Thus, we create a corrected flux-limited sample; for each SDSS exponential (fracDeV $<0.1$ ) galaxy we ask not simply whether it is above our flux limit, but whether it would be above our flux limit if it were edge-on. If an SDSS exponential galaxy has $q=0.2$, we assume that it is already edge-on. If it has an observed apparent axis ratio $q_{\mathrm{obs}}>0.2$, we compute its edgeon flux to be

$$
\begin{aligned}
m_{r}(\text { edge-on }) & =m_{r}(\text { observed })+\Delta M_{r}(q=0.2)-\Delta M_{r}\left(q_{\text {obs }}\right) \\
& =m_{r}(\text { observed })+1.27\left[(\log 0.2)^{2}-\left(\log q_{\text {obs }}\right)^{2}\right] .
\end{aligned}
$$

We create our corrected flux-limited sample by demanding that this computed edge-on flux be greater than $m_{r}=17.77$, the completeness limit of the SDSS spectroscopic survey for galaxies. To ensure that our inclination-corrected sample contains only the luminous, nearby galaxies for which our $\Delta M_{r}(q)$ correction was computed, we add the addition restrictions $M_{r}^{f}<-19.4$ and $z<0.06$.

The distribution of the apparent axis ratio $q$ for the $n=16,155$ exponential galaxies in our inclination-corrected sample is shown in Figure 11 (top). The distribution shown as the solid line is estimated using a nonparametric kernel technique (Vio et al. 1994; Tremblay \& Merritt 1995). To ensure that our estimate of $f(q)$ is smooth, we use a Gaussian kernel. The kernel width is chosen using the standard adaptive two-stage estimator of Abramson (1982). To ensure that our estimate of $f(q)$ is zero for $q<0$ and $q>1$, we impose reflective boundary conditions at $q=0$ and 1 . The dashed lines in Figure 11 indicate the $98 \%$ error intervals on $f(q)$ found by bootstrap resampling. At every value of $q, 1 \%$ of the bootstrap estimates lie above the upper dashed line, and $1 \%$ lie below the lower dashed line. For purposes of comparison, the green dot-dashed line in Figure 11 (top) is the distribution of $q$ that we would have found by ignoring inclination corrections.
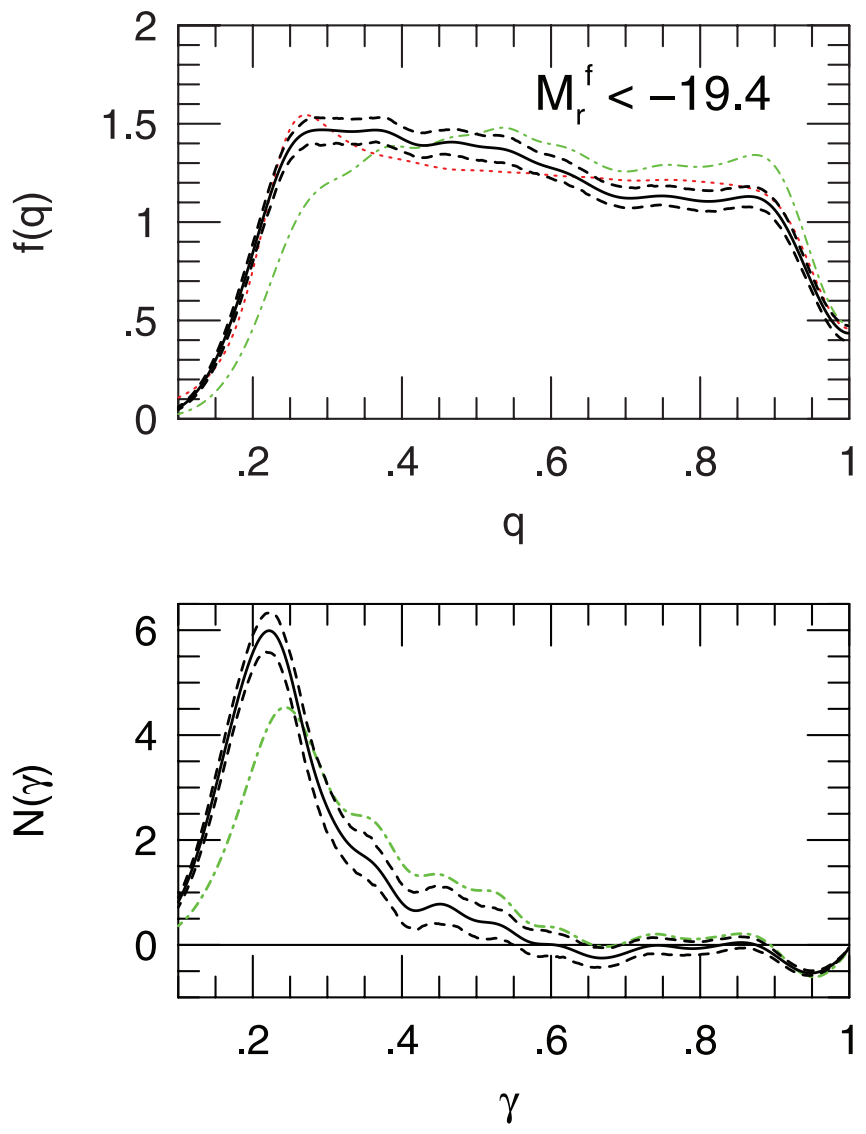

FIG. 11.-Top: Distribution of apparent axis ratios for the inclination-corrected sample of SDSS exponential galaxies. The solid line is the best fit: the dashed lines show the $98 \%$ confidence interval estimated from bootstrap resampling. The green dot-dashed line is the distribution found without including inclination corrections. The red dotted line is the distribution of $q$ yielded by the best-fitting parametric distribution of elliptical disks (eqs. [17] and [18]). Bottom: Distribution of intrinsic axis ratios, assuming galaxies are randomly inclined oblate spheroids. The solid line is the inversion of the best fit for $f(q)$; the dashed lines are the $98 \%$ confidence interval from bootstrap resampling. The green dot-dashed line is the distribution found without including inclination corrections.

Having a smooth estimate of $f(q)$, the distribution of apparent axis ratios, allows us to compute $N(\gamma)$, the distribution of intrinsic axis ratios, given the assumption that all galaxies are oblate or prolate. If the disk galaxies are assumed to be oblate, the relation between $f(q)$ and $N(\gamma)$ is

$$
f(q)=\int_{0}^{q} P_{\mathrm{obl}}(q \mid \gamma) N(\gamma) d \gamma
$$

where $P_{\mathrm{obl}}(q \mid \gamma) d q$ is the conditional probability that an oblate spheroid with an intrinsic short-to-long axis ratio $\gamma$ has an observed apparent axis ratio in the range $q \rightarrow q+d q$, averaged over all viewing angles. The numerical value of $P_{\mathrm{obl}}$ is (Sandage et al. 1970)

$$
P_{\mathrm{obl}}(q \mid \gamma)=\frac{q}{\left(1-\gamma^{2}\right)^{1 / 2}\left(q^{2}-\gamma^{2}\right)^{1 / 2}}
$$

if $\gamma \leq q \leq 1$, and $P_{\text {obl }}=0$ otherwise. Equation (15) is a Volterra equation of the first kind; in its discretized form, it can be inverted by a process of forward substitution to find $N(\gamma)$ given $f(q)$ (see Vincent \& Ryden 2005 for numerical details).

In Figure 11 (bottom), the solid line indicates the estimate of $N(\gamma)$ found by inverting the best estimate of $f(q)$; the dashed 
lines are the $98 \%$ confidence intervals found from the inversion of the bootstrap estimates of $f(q)$. The green dot-dashed line is the estimate of $N(\gamma)$ we would have found if we had omitted inclination corrections. For the inclination-corrected sample, the most probable value of $\gamma$, given the oblate hypothesis, is $\gamma=0.22$. For comparison to these $r$-band results, an inclination-corrected sample of spiral galaxies from the 2MASS Large Galaxy Atlas has a most probable thickness of $\gamma_{B}=0.17$ in the $B$ band and $\gamma_{K}=0.25$ in the $K_{s}$ band (Ryden 2006). Our estimate of $N(\gamma)$ in Figure 11 is negative for large values of $\gamma$. The $98 \%$ confidence interval is negative for $\gamma \geq 0.89$; that is, fewer than $1 \%$ of our bootstrap resamplings give $N(\gamma)>0$ in this interval. This unphysical result permits us to reject the hypothesis of perfect oblateness at the $99 \%$ (one sided) confidence level.

We can approximate a galaxy in our sample not as an oblate spheroid, but as a triaxial ellipsoid, with axis lengths $a \geq b \geq c$. The shape can then be expressed in terms of two parameters, which we choose to be the short-to-long axis ratio, $\gamma \equiv c / a$, and the disk ellipticity, $\epsilon \equiv 1-b / a$. Once we permit nonzero values of $\epsilon$, we can no longer use the observed distribution $f(q)$ to uniquely determine the intrinsic distribution $N(\gamma, \epsilon)$. However, we still can do parametric fits to the distribution of shapes. It is found that a useful parameterization is $N(\gamma, \epsilon)=N_{\gamma}(\gamma) N_{\epsilon}(\epsilon)$, with $N_{\gamma}$ being a Gaussian,

$$
N_{\gamma}(\gamma) \propto \exp \left[-\frac{\left(\gamma-\mu_{\gamma}\right)^{2}}{2 \sigma_{\gamma}^{2}}\right],
$$

and $N_{\epsilon}$ being a lognormal distribution (Andersen et al. 2001; Ryden 2004, 2006),

$$
N_{\epsilon}(\epsilon) \propto \frac{1}{\epsilon} \exp \left[-\frac{(\ln \epsilon-\mu)^{2}}{2 \sigma^{2}}\right] .
$$

The best values of $\mu_{\gamma}, \sigma_{\gamma}, \mu$, and $\sigma$ were determined by a $\chi^{2}$ fit to the binned distribution of $q$ for the $n=16,155$ exponential galaxies in our inclination-corrected sample. The bin width chosen was $d q=0.01$. After selecting values of $\mu_{\gamma}, \sigma_{\gamma}, \mu$, and $\gamma$, we randomly chose $n$ values of $\gamma$ and of $\epsilon$ from the distributions of equations (17) and (18). For each $(\gamma, \epsilon)$ pair, a random viewing angle was chosen, and the resulting apparent axis ratio was computed (Binney 1978). The model axis ratios were then binned in the same way as the actual, observed axis ratios. Repeating this procedure 400 times for each $\left(\mu_{\gamma}, \sigma_{\gamma}, \mu, \sigma\right)$ set, we calculated the mean and standard deviation for the number of model galaxies in each $q$ bin, and computed a $\chi^{2}$ probability for that particular set of parameters. The best fit we found was $\left(\mu_{\gamma}, \sigma_{\gamma}\right)=$ $(0.216,0.067)$ for the distribution of disk thicknesses, with $(\mu, \sigma)=(-2.56,0.91)$ for the distribution of the natural logarithm of the ellipticity. The $\chi^{2}$ probability yielded by this set of parameters is $P \sim 2 \times 10^{-7}$. In Figure 11 (top), the dotted red line shows the distribution of $q$ expected from this set of parameters, smoothed with the same kernel width as the real data (solid black line). If we had neglected the inclination corrections to our sample, we would have found a best fit $\left(\mu_{\gamma}, \sigma_{\gamma}\right)=$ $(0.272,0.102)$ and $(\mu, \gamma)=(-2.68,0.69)$, significantly overestimating both the mean and the standard deviation of the disk thickness $\gamma$.

The distribution of ellipticity of our best-fitting parametric model, $\ln \epsilon=-2.56 \pm 0.91$, implies a modal ellipticity $\epsilon_{\bmod }=$ 0.033 , a median ellipticity $\epsilon_{\text {med }}=0.077$ and a mean ellipticity $\epsilon_{\mathrm{ave}}=0.11$. This distribution of thicknesses is consistent with the implied ellipticity of an inclination-corrected sample of spiral galaxies from the 2MASS Large Galaxy Atlas (Ryden 2006), if the shape parameter for the 2MASS spirals is the axis ratio of the 25 mag $\operatorname{arcsec}^{-2}$ isophote. If the potential ellipticity equals the disk ellipticity, then $\ln \epsilon=-2.56 \pm 0.91$ implies roughly $1 \mathrm{mag}$ of scatter in the Tully-Fisher relation (Tully \& Fisher 1977).

\section{DISCUSSION}

We have selected a population of galaxies from the Sloan Digital Sky Survey which are at low redshift $(z<0.06)$, which are relatively luminous $\left(M_{r} \leq-19\right)$, and which are well described by an exponential surface brightness profile (fracDeV $<$ 0.1 ). As we have shown, the properties of these galaxies are consistent with their being a population of slightly elliptical disks containing dust. The median dimensionless disk thickness for these galaxies in the $r$ band is $\gamma \approx 0.22$; the median disk ellipticity is $\epsilon \approx 0.08$.

By fitting the luminosity function for galaxies with different apparent axis ratio $q$, we found that the apparent dimming $\Delta M_{r}$ is not linearly proportional to $\log q$, but instead is much better fitted by $\Delta M_{r} \propto(\log q)^{2}$. The dependence of dimming on inclination is a valuable clue to the dust properties within disk galaxies. If certain simplifying assumptions are made, the expected attenuation as a function of inclination can be computed for model galaxies. For instance, Ferrara et al. (1999) assumed that dust had either the extinction curve found for Milky Way dust or for Small Magellanic Cloud dust (Gordon et al. 1997). They assumed that disks were perfectly axisymmetric, with a horizontal scale length $r=4 \mathrm{kpc}$ that was the same for both stars and dust. The scale height of the stars was assumed to be $z_{\star}=0.35$, but the scale height of the dust was allowed to vary. Ferrara et al. (1999) found that dimming in the $B$ and $I$ bands were proportional to $\log q$ only when the dust scale height was greater than that of the stars. However, observation of nearby edge-on disk galaxies (Xilouris et al. 1999) indicates that the dust scale height is about half the star scale height.

The results of more physically realistic hydrodynamic simulations also indicate that $\Delta M$ is not linearly proportional to $\log q$. Rocha et al. (2008) did a study of internal extinction in dusty disk galaxies, using the smoothed particle hydrodynamics (SPH) simulations described in detail in Cox et al. (2006) and Jonsson et al. (2006). Briefly, the SPH simulations contain a rotationally supported disk of gas and stars, a spheroidal bulge, and a dark matter halo. Numerical schemes for radiative cooling, supernova feedback, and metal enrichment are included in the models, and a star formation prescription based on a Schmidt (1959) law is assumed. The metallicity gradients of the model galaxies, given in Table 2 of Rocha et al. (2008) range from approximately -0.02 to $-0.06 \mathrm{dex} \mathrm{kpc}^{-1}$, and are chosen to match the observational result of Xilouris et al. (1999) that bright spiral galaxies have a dust scale length that is 1.4 times the gas scale length in the disk. The dust model used was the $R=3.1$ Milky Way model of Weingartner \& Draine (2001). The observable properties of the model galaxies were determined using SUNRISE, a Monte Carlo radiative transfer code (Jonsson 2006). Since SUNRISE provides a full spectral energy distribution for a given model viewed from a given inclination, an absolute magnitude for any standard filter can be computed. Rocha et al. (2008) published results for the $U, B, V, R, I, J, H$, and $K$ filters; M. Rocha (2008, private communication) has supplied us with the equivalent results in the Sloan $r$ band.

The model galaxies studied by Rocha et al. (2008) fall into two different series. The "S series" were modeled to mimic the properties of gas-rich late-type spiral galaxies. The Sc model represents a very gas-rich spiral, with $f_{g}$, the fraction of the 
baryonic matter in the form of gas, having the value $f_{g}=0.69$. The $r$-band face-on absolute magnitude of the Sc model is $M_{r}^{f}=$ -21.95 . The three Sbc models represent slightly less gas-rich galaxies, with $f_{g}=0.52$; they differ in their disk scale length $R_{d}$ and absolute magnitude, ranging from $R_{d}=4.0 \mathrm{kpc}$ and $M_{r}^{f}=$ -20.97 for the Sbc - model, through $R_{d}=5.5 \mathrm{kpc}$ and $M_{r}^{f}=$ -21.85 for the Sbc model, to $R_{d}=7.0 \mathrm{kpc}$ and $M_{r}^{f}=-22.19$ for the Sbc+ model. The second series of model galaxies, called the "G series," have significantly less gas than the $\mathrm{S}$ series, and cover a wider range of luminosities. The faintest of the $\mathrm{G}$ series, called G0, falls far below the $M_{r} \sim-19$ threshold at which exponential galaxies show the characteristic of thin dusty disks. The G1 model has $f_{g}=0.29$ and $M_{r}^{f}=-18.79$; the G2 model has $f_{g}=0.23$ and $M_{r}^{f}=-20.07$; the G3 model has $f_{g}=0.20$ and $M_{r}^{f}=-21.32$.

Figure 7 shows the dimming for the $\mathrm{S}$ series and $\mathrm{G}$ series models of Rocha et al. (2008) viewed at inclination $i=35^{\circ}$, $51^{\circ}, 63^{\circ}, 74^{\circ}$, and $85^{\circ}$. All the models show a quadratic, rather than linear, dependence on $\log q$, in agreement with our results. If we look at the amplitude of the dimming, the best fit to our observational results is given by the G3 model, a luminous galaxy with a modest gas fraction. The models $\mathrm{Sbc}+, \mathrm{Sc}$, and $\mathrm{Sbc}$, which represent highly luminous $\left(M_{r}^{f} \sim-22\right)$ galaxies with a high gas fraction $\left(f_{b} \geq 0.52\right)$, show more dimming at a given $q$ than we find by our technique, which is designed to find the dimming for nearby galaxies with $M_{r}^{f} \sim M_{r}^{*} \sim-20$. The model G1, which represents a relatively low luminosity $\left(M_{r}^{f} \sim-18.8\right)$ galaxy with a modest gas fraction $\left(f_{b} \sim 0.3\right)$, shows less dimming than we find with our technique.

In our sample of exponential galaxies from the Sloan Digital Sky Survey, once the absolute magnitude of a galaxy is corrected for the inclination-dependent dimming, the mean $u-r$ color observed is linearly dependent on the corrected $M_{r}^{f}$. For nearly face-on galaxies, with $q>0.9$, the dependence of $u-r$ on $M_{r}^{f}$ is relatively weak. We find $b=-0.096$; that is, less than $0.1 \mathrm{mag}$ of reddening in $u-r$ for each magnitude brighter in $M_{r}^{f}$. For the edge-on galaxies, with $q<0.3$, the dependence of $u-r$ on $M_{r}^{f}$ is much stronger, with $b=-0.265$. The mean color-absolute magnitude dependence is a manifestation of the metallicityluminosity dependence. High-metallicity galaxies have both redder stellar populations and higher dust contents. For faceon exponential galaxies, the dust effects are minimized, and we see the effect of metallicity on stellar populations. For edge-on exponential galaxies, we see, in addition, the effect of metallicity on the dust content. Exponential galaxies with $M_{r}^{f} \sim-21.5$ have a typical color $\langle u-r\rangle \sim 1.8$ when seen face-on, placing them at the tip of the blue sequence in a color-magnitude diagram. However, the same bright exponential galaxies when seen edgeon will have $M_{r} \sim-21$ and $\langle u-r\rangle \sim 2.5$, a degree of reddening that smuggles them into the red sequence, as usually defined.

Miguel Rocha kindly translated the results of Rocha et al. (2008) into the Sloan passbands; Joel Primack provided helpful comments. Funding for the SDSS and SDSS-II has been provided by the Alfred P. Sloan Foundation, the Participating Institutions, the National Science Foundation, the US Department of Energy, the National Aeronautics and Space Administration, the Japanese Monbukagakusho, the Max Planck Society, and the Higher Education Funding Council for England. The SDSS Web site is at http://www.sdss.org/. The SDSS is managed by the Astrophysical Research Consortium (ARC) for the Participating Institutions. The Participating Institutions are the American Museum of Natural History, Astrophysical Institute Potsdam, University of Basel, University of Cambridge, Case Western Reserve University, University of Chicago, Drexel University, Fermilab, the Institute for Advanced Study, the Japan Participation Group, Johns Hopkins University, the Joint Institute for Nuclear Astrophysics, the Kavli Institute for Particle Astrophysics and Cosmology, the Korean Scientist Group, the Chinese Academy of Sciences (LAMOST), Los Alamos National Laboratory, the Max Planck Institute for Astronomy (MPIA), the Max Planck Institute for Astrophysics (MPA), New Mexico State University, The Ohio State University, University of Pittsburgh, University of Portsmouth, Princeton University, the United States Naval Observatory, and the University of Washington.
Abramson, I. S. 1982, Ann. Stat., 10, 1217

Adelman-McCarthy, J. K., et al. 2008, ApJS, 175, 297

Alam, S. M. K., \& Ryden, B. S. 2002, ApJ, 570, 610

Andersen, D. R., Bershady, M. A., Sparke, L. S., Gallagher, J. S., \& Wilcots, E. M. 2001, ApJ, 551, L131

Baldry, I. K., Glazebrook, K., Brinkmann, J., Ivezic, Z., Lupton, R. H., Nichol, R. C., \& Szalay, A. S. 2004, ApJ, 600, 681

Ball, N. M., Loveday, J., Brunner, R. J., Baldry, I. K., \& Brinkmann, J. 2006, MNRAS, 373, 845

Ball, N. M., Loveday, J., Fukugita, M., Nakamura, O., Brinkmann, J., \& Brunner, R. J. 2004, MNRAS, 348, 1038

Bell, E. F., \& de Jong, R. S. 2000, MNRAS, 312, 497

Binney, J. 1978, MNRAS, 183, 779

Binney, J., \& de Vaucouleurs, G. 1981, MNRAS, 194, 679

Blanton, M. R., et al. 2003, ApJ, 594, 186

Bottinelli, L., Gouguenheim, L., Paturel, G., \& Teerikorpi, P. 1995, A\&A, 296, 64

Chang, R., Shen, S., Hou, J., Shu, C., \& Shao, Z. 2006, MNRAS, 372, 199

Cox, T. J., Jonsson, P., Primack, J. R., \& Somerville, R. S. 2006, MNRAS, 373, 1013

de Vaucouleurs, G. 1948, Ann. d'Astrophys., 11, 247

de Vaucouleurs, G., de Vaucouleurs, A., Corwin, H. G., Jr., Buta, R. J., Paturel, G., \& Fouque, P. 1991, Third Reference Catalogue of Bright Galaxies (New York: Springer) (RC3)

Fasano, G., Amico, P., Bertola, F., Vio, R., \& Zeilinger, W. W. 1993, MNRAS, 262, 109

Ferrara, A., Bianchi, S., Cimatti, A., \& Giovanardi, C. 1999, ApJS, 123, 437

Fukugita, M., Shimasaku, K., \& Ichikawa, T. 1995, PASP, 107, 945

Gordon, K. D., Calzetti, D., \& Witt, A. N. 1997, ApJ, 487, 625

Grosbøl, P. J. 1985, A\&AS, 60, 261
REFERENCES

Holmberg, E. 1958, Meddelande Från Lunds Astron. Obs. Ser. II, 136, 1

Hubble, E. P. 1926, ApJ, 64, 321

Jonsson, P. 2006, MNRAS, 372, 2

Jonsson, P., Cox, T. J., Primack, J. R., \& Somerville, R. S. 2006, ApJ, 637, 255 Lambas, D. G., Maddox, S. J., \& Loveday, J. 1992, MNRAS, 258, 404

MacArthur, L. A., Courteau, S., Bell, E., \& Holtzman, J. A. 2004, ApJS, 152, 175

Masters, K. L., Giovanelli, R., \& Haynes, M. P. 2003, AJ, 126, 158

Rocha, M., Jonsson, P., Primack, J. R., \& Cox, T. J. 2008, MNRAS, 383, 1281

Ryden, B. S. 1992, ApJ, 396, 445 2004, ApJ, 601, 214 2006, ApJ, 641, 773

Sandage, A., Freeman, K. C., \& Stokes, N. R. 1970, ApJ, 160, 831

Schechter, P. 1976, ApJ, 203, 297

Schmidt, M. 1959, ApJ, 129, 243

Sérsic, J. L. 1968, Atlas de Galaxias Australes (Cordoba: Obs. Astron.)

Shao, Z., Xiao, Q., Shen, S., Mo, H. J., Xia, X., \& Deng, Z. 2007, ApJ, 659, 1159

Smith, J. A., et al. 2002, AJ, 123, 2121

Strateva, I. 2001, AJ, 122, 1861

Tremblay, B., \& Merritt, D. 1995, AJ, 110, 1039

Tully, R. B., \& Fisher, J. R. 1977, A\&A, 54, 661

Tully, R. B., Pierce, M. J., Huang, J.-S., Saunders, W., Verheijen, M. A. W., \&

Witchalls, P. L. 1998, AJ, 115, 2264

Valentijn, E. A. 1990, Nature, 346, 153

Vincent, R. A., \& Ryden, B. S. 2005, ApJ, 623, 137

Vio, R., Fasano, G., Lazzarin, M., \& Lessi, O. 1994, A\&A, 289, 640

Weingartner, J. C., \& Draine, B. T. 2001, ApJ, 548, 296

Xilouris, E. M., Byun, Y. I., Kylafis, N. D., Paleolougou, E. V., \& Papamastorakis, J. 1999, A\&A, 344, 868

York, D. G., et al. 2000, AJ, 120, 1579 